\title{
CAPÍTULO 02: CAMU-CAMU (MYRCIARIA DUBIA (H.B.K.) MC VAUGH): PADRÃO DE IDENTIDADE E QUALIDADE, COMPOSTOS FENÓLICOS, CAPACIDADE REDUTORA E ANTIOXIDANTE DA POLPA
}

\author{
CHAPTER 02: CAMU-CAMU (MYRCIARIA DUBIA (H.B.K.) MC VAUGH): \\ IDENTITY AND QUALITY, PHENOLIC COMPOUNDS, REDUCING AND \\ ANTIOXIDANT CAPACITY OF PULP
}

\begin{abstract}
Carolina Kato Prado ${ }^{1}$; Jéssica Barrionuevo Ressutte ${ }^{2}$; Natália Vicente Rezende Mudenuti ${ }^{3}$; Tiago Bervelieri Madeira $^{4}$; Wilma Aparecida Spinosa ${ }^{5}$
\end{abstract}

\begin{abstract}
Resumo
$\mathrm{O}$ camu-camu é conhecido pelo seu alto teor de vitamina $\mathrm{C}$ e compostos bioativos, porém, o consumo desse fruto é limitado devido ao seu sabor ácido e adstringente. Estudos que possam caracterizar o camu-camu são relevantes, pois podem fornecer informações úteis para um melhor aproveitamento tecnológico desse fruto pela indústria. Nesse contexto, o objetivo deste trabalho foi caracterizar a polpa do camu-camu quanto ao seu padrão de identidade e qualidade. Para isso foram realizadas análises físico-químicas, ensaios de capacidade antioxidante e redutora e compostos fenólicos. O camu-camu apresentou umidade de 92,77 g/100g, vitamina $\mathrm{C}$ de $2,24 \mathrm{~g} / 100 \mathrm{~g}$ e capacidade redutora de 2,60 g EAG/100g. O pH foi de 2,93 e o teor de açúcares totais de $8,00 \mathrm{~g} / 100 \mathrm{~g}$. Os compostos fenólicos presentes no camu-camu foram ácido nicotínico, ácido gálico, ácido protocatecuico, teobromina, epigalocatequina, ácido hidroxibenzóico, catequina, ácido clorogênico, cafeína, epicatequina, ácido p-cumárico, ácido sinápico, quercetina, rutina, miricetina e kaempferol. A capacidade antioxidante para os métodos avaliados por DPPH, ABTS e FRAP foram de 57,30 $\mu$ mol trolox/g, 167,36 $\mu \mathrm{mol}$ trolox/g e 1133,03 $\mu \mathrm{mol} \mathrm{Fe}^{2} \mathrm{SO} 4 / \mathrm{g}$, respectivamente. A partir dos resultados obtidos pode-se inferir que o camu-camu tem potencial para uso na indústria de alimentos como importante fonte de compostos bioativos.
\end{abstract}

Palavras-Chaves: Polpa de camu-camu, compostos bioativos, antioxidantes, ácido ascórbico, polifenóis.

\begin{abstract}
Camu-camu is known for its high content of vitamin $\mathrm{C}$ and bioactive compounds, however, the consumption of this fruit is limited due to its acidic and astringent flavor. Studies that can characterize camu-camu are relevant, because they can provide useful information for a better technological use of this fruit by the industry. In this context, the objective of this work was to characterize the camu-camu pulp in terms of its identity and quality standard. For this, physicochemical analyzes, tests of antioxidant and reducing capacity and phenolic compounds were carried out. The camu-camu showed $92.77 \mathrm{~g} / 100 \mathrm{~g}$ moisture, $2.24 \mathrm{~g} / 100 \mathrm{~g}$ vitamin $\mathrm{C}$ and $2.60 \mathrm{~g}$ EAG/100g reducing capacity. The $\mathrm{pH}$ was 2.93 and the total sugar content was 8.00 $\mathrm{g} / 100 \mathrm{~g}$. The phenolic compounds present in camu-camu were nicotinic acid, gallic acid, protocatechuic acid, theobromine, epigallocatechin, hydroxybenzoic acid, catechin, chlorogenic acid, caffeine, epicatechin, p-cumaric acid, synapic acid, quercetin, rutin, myricetin

\footnotetext{
${ }^{1}$ Doutora em Ciência de Alimentos, Universidade Estadual de Londrina, carolkprado@ gmail.com

${ }^{2}$ Doutoranda em Ciência de Alimentos, Universidade Estadual de Londrina, jessicaressutte@ gmail.com

${ }^{3}$ Doutora em Ciência de Alimentos, Universidade Estadual de Londrina, natvr9@ hotmail.com

${ }^{4}$ Doutor em Química, Universidade Estadual de Londrina, madeiratb@gmail.com

${ }^{5}$ Doutora em Ciência de Alimentos, Universidade Estadual de Londrina, wilma.spinosa@uel.br
} 
and kaempferol. The antioxidant capacity for the methods evaluated by DPPH, ABTS and FRAP were $57.30 \mu \mathrm{mol}$ trolox/g, $167.36 \mu \mathrm{mol}$ trolox/g and $1133.03 \mu \mathrm{mol} \mathrm{Fe}^{2} \mathrm{SO} 4 / \mathrm{g}$, respectively. From the results obtained, it can be inferred that the camu-camu has potential for use in the food industry as an important source of bioactive compounds.

Keywords: Camu-camu pulp, bioactive compounds, antioxidants, ascorbic acid, polyphenols.

\section{Introdução}

O camu-camu (Myrciaria dubia (H. B. K.) McVaugh), também chamado de caçari ou araçá-d'água, é um fruto esférico, com superfície lisa e coloração vermelho-escuro, conhecido por possuir elevada umidade e acidez. A planta de cam-camu é pertencente à família Myrtaceae, sendo encontrada dispersa em quase toda a Amazônia, nas margens dos rios e lagos. O camucamuzeiro leva de 3 ou 4 anos para começar a produzir frutos, após seu plantio definitivo. A frutificação dessa planta ocorre entre os meses de novembro e março (INPA, 2018; RIBEIRO; MOTA; CORRÊA, 2002). Atualmente o Peru é o país líder de produção e comercialização, porém, países vizinhos como a Colômbia, Brasil e Venezuela têm expandido o cultivo do camu-camu (KANESHIMA et al., 2017).

No Brasil, o camu-camu é encontrado desde o litoral Atlântico no estado do Pará até a região Amazônia do Tocantins, sendo cultivado principalmente nos estados do Maranhão, Amapá, Pará, Roraima, Rondônia e Tocantins (EMBRAPA, 2012, SOUZA-FILHO et al., 2002). Esse fruto tem sido estudado pelo Instituto Nacional de Pesquisas da Amazônia (INPA), desde 1980 e pela Empresa Brasileira de Pesquisa Agropecuária (EMBRAPA) desde 2007.

Devido à elevada acidez da polpa, o camu-camu é mais utilizado no preparo de sucos, concentrado para preparo de outros produtos alimentícios, e para preparo de cápsulas de vitamina C (AZEVÊDO et al., 2015). Entretanto um dos fatores que contribuem para a restrição do seu consumo são o sabor muito ácido da polpa e o amargor da casca, levando à necessidade de pesquisas para seu melhor aproveitamento. Uma das alternativas para a utilização deste fruto é na forma de polpa congelada (MAEDA et al., 2006). A polpa congelada apresenta vantagens como poder ser transportada para outros locais e poder ser utilizada como ingrediente para a produção de outros alimentos, contribuindo dessa forma, com a conservação do fruto, além de aumentar a renda dos produtores e flexibilizar a sua aplicação para produção de alimentos (CONCEIÇÃO et al., 2020).

As propriedades do camu-camu despertam interesses econômicos e científicos das indústrias farmacêuticas, apresentando um grande potencial econômico capaz de colocá-lo no mesmo nível de importância de outras frutas tradicionais da região amazônica, como o açaí e cupuaçu (NASCIMENTO et al., 2013) e por isso o camu-camu tem sido exportado para Japão 
e Estados Unidos em grande escala (AGUIAR; SOUZA, 2015).

A Myrciaria dubia é conhecida pelo seu alto teor de ácido ascórbico, que comparado ao da acerola é aproximadamente 20 vezes maior (VIDIGAL et al., 2011). Os frutos de camucamu também apresentam vários tipos de aminoácidos e quantidades consideráveis de minerais (AGUIAR; SOUZA, 2018; CUNHA-SANTOS et al., 2019; FUJITA et al., 2017). Ademais, o camu-camu apresenta altos teores de de compostos fenólicos, com efeitos neuroprotetores (AZEVÊDO et al., 2015), hepatoprotetores (AKACHI et al., 2010), antimutagênicos (AZEVEDO et al., 2019), hipoglicemiantes (BALISTEIRO et al., 2017) e anti-inflamatórios (INOUE et al., 2008).

Tanto a vitamina $\mathrm{C}$ quanto os compostos fenólicos são classificados como antioxidantes não-enzimáticos, sendo eles naturais. O ácido ascórbico é um sequestrador de radicais livres solúvel em água, que doa um elétron para o radical lipídico e se torna radical ascorbato, impedindo a reação em cadeia da peroxidação lipídica (NIMSE; PAL, 2015). Os compostos fenólicos por sua vez podem atuar impedindo a oxidação de diversas formas: pela ligação a metais, pelo sequestro de espécies reativas de oxigênio (ERO), pela produção de óxido nítrico e modulação de enzimas envolvidas na produção de ERO (BARADARAN; NASRI; RAFIEIAN-KOPAEI, 2014).

O estresse oxidativo está relacionado a uma série de doenças e também ao processo de envelhecimento, sendo resultado de um desequilíbrio entre a produção de ERO e as defesas antioxidantes do organismo (KALLAUR; OLIVEIRA; REICHE, 2014). As frutas e vegetais possuem grande variedade de antioxidantes que protegem as células dos danos oxidativos e consequentemente diminuem o risco de doenças crônicas, já que atuam impedindo ou retardando a oxidação de substâncias (URQUIZA-MARTÍNEZ; NAVARRO, 2016). Por isso, há uma grande procura por produtos naturais que possam ter uma potencial contribuição no tratamento de doenças (LANGLEY et al., 2015).

Sendo assim, o objetivo desse trabalho foi caracterizar o camu-camu em relação às suas composições físico-química e de compostos fenólicos e avaliar sua capacidade antioxidante e redutora visando seu potencial econômico e aplicação na indústria de alimentos.

\section{Material e Métodos}

\section{Material}

A polpa congelada de camu-camu foi doada pela empresa PolpaSul. Os frutos com grau de maturação maduro, de coloração vermelha, adquiridos pela empresa foram provenientes de árvores cultivadas a beira do rio Madeira na região Amazônica. Após o despolpe, o produto, 
isento de qualquer aditivo, foi envasado em embalagens plásticas individuais com capacidade para 100 gramas e congelado para posteriores análises físico-químicas, de capacidades redutora e antioxidante.

\section{Análises físico-químicas}

Foram realizadas análises de $\mathrm{pH}$, sólidos solúveis, acidez titulável, gorduras totais, proteínas totais, açúcares totais, umidade e ácido ascórbico.

O pH (pHmetro PG2000, Gehaka®, Brasil), acidez total em ácido cítrico, sólidos solúveis em ${ }^{\circ}$ Brix (Refratômetro - RM40, Mettler Toledo®, USA) e vitamina C foram obtidos de acordo com metodologia da IAL (2008), em que a acidez total foi expressa em g/100g e a vitamina $\mathrm{C}$ em $\mathrm{mg} / 100 \mathrm{~g}$. Para as análises de umidade, proteínas totais, gorduras totais e açúcares totais foram utilizadas metodologias de acordo com a AOAC (2012) e os resultados foram expressos em $\mathrm{g} / 100 \mathrm{~g}$.

Determinação de compostos fenólicos, análises de capacidade redutora e capacidade antioxidante

Para essas análises foi feito um extrato a partir da polpa congelada de camu-camu. $\mathrm{O}$ extrato foi obtido de acordo com metodologia de Rufino et al. (2011), utilizando-se de duas extrações de $5 \mathrm{~g}$ de amostra, a primeira com uma mistura de água e metanol e a segunda com acetona e água.

\section{Capacidade Redutora}

A determinação da capacidade redutora foi realizada pelo método de Folin-Ciocalteau Singleton e Rossi (1965), modificado por Reynertson et al. (2008). A curva padrão de solução de ácido gálico foi obtida em duplicata com concentrações de 50, 100, 150, 180, 200 e 250 $\mu \mathrm{g} / \mathrm{mL}$ e a absorbância foi lida a $765 \mathrm{~nm}$.

\section{Capacidade Antioxidante}

As análises para determinação de capacidade antioxidante foram realizadas utilizando três métodos: DPPH (2,2-difenil-1-Picrilhidrazil), ABTS (ácido 2,2'-azinobis (3eilbenzotiazolínico-6-sulfônico)) e FRAP (Poder antioxidante de redução do íon Ferro), de acordo com Rufino et al. (2010).

\section{$D P P H$}


Nessa análise 3,9 mL de solução DPPH a 0,06 mmol/L foram adicionados a $100 \mu \mathrm{l}$ de extrato de camu-camu. A absorbância foi lida a $515 \mathrm{~nm}$ a intervalos de 1 minuto nos primeiros 10 minutos e em intervalos de 5 minutos até a estabilização. A curva padrão de solução de Trolox (ácido 6-hidroxi-2,5,7,8-tetrametilcromano-2-caboxílico) foi obtida a partir de uma solução-mãe a $250 \mu \mathrm{g} / \mathrm{mL}$ diluída nas concentrações de 25, 50, 100, 150 e $200 \mu \mathrm{g} / \mathrm{mL}$.

\section{ABTS}

A determinação de capacidade antioxidante pelo método ABTS foi feita adicionandose $30 \mu \mathrm{L}$ do extrato de camu-camu diluído a $5000 \mu \mathrm{g} / \mathrm{mL}$ a $3 \mathrm{~mL}$ de solução de trabalho ABTS, que 6 minutos após agitação, a absorbância foi medida a $734 \mathrm{~nm}$. A curva padrão foi obtida por meio de diluições a $0.3,0.5,0.7,1.0,1.5,1.8$ e $2.0 \mathrm{mM}$ a partir de uma solução estoque de Trolox a $8 \mathrm{mM}$.

\section{FRAP}

Para essa determinação $0,9 \mathrm{~mL}$ do reagente FRAP a $37^{\circ} \mathrm{C}$ foram misturados a $30 \mu \mathrm{L}$ de amostra e $90 \mu \mathrm{L}$ de água destilada, sendo lida a absorbância a 595 nm, após 30 minutos a temperatura de $37^{\circ} \mathrm{C}$. A curva padrão foi feita utilizando as concentrações de 50, 100, 200, 300, 400,500 e $600 \mu \mathrm{g} / \mathrm{mL}$.

Quantificação de compostos fenólicos por Cromatografia líquida de ultra eficiência acoplado a detector de massas (UPLC-MS/M)

Para essa análise, $1 \mathrm{~mL}$ do extrato de camu-camu foi seco em fluxo de nitrogênio e ressuspendido em $1 \mathrm{~mL}$ de Metanol. Para determinação de compostos fenólicos, utilizou-se um cromatógrafo líquido (UPLC - Ultra Performance Liquid Chromatography) (Acquity UPLC, Waters, MA, EUA) acoplado a um detector de massas (Acquity Tandem Quadrupolo, Waters, MA, EUA) no modo ionização positivo, com temperatura do gás de dessolvatação (Nitrogênio) de $500^{\circ} \mathrm{C}$ a uma vazão de $900 \mathrm{~L} / \mathrm{h}$, gás do cone mantido à mesma temperatura na vazão de 20 $\mathrm{L} / \mathrm{h}$. A temperatura de fonte de ionização foi de $120^{\circ} \mathrm{C}$. As voltagens do cone amostral e do extrator foram otimizadas para cada composto e a voltagem do capilar foi de 3,5 KV. Para a separação dos compostos fenólicos foi utilizada uma coluna Waters BEH C8 com dimensões de 2,1 x $50 \mathrm{~mm}$ e partícula de 1,7 $\mu \mathrm{m}$. As condições cromatográficas foram: fase móvel A composta por água e $0,1 \%$ de ácido fórmico e fase móvel B composta por metanol e $0,1 \%$ de ácido fórmico. De 0 a 0,4 minutos o gradiente de fase móvel utilizado foi de 95\% (A) e 5\% (B), posteriormente a fase (B) teve sua proporção aumentada linearmente até $95 \%$ no tempo de 15,40 
minutos e mantida na mesma proporção até 18,40 minutos. No tempo de 18,41 minutos, retornaram-se as proporções das fases às condições iniciais até 20 minutos. O volume de injeção da amostra foi de $10 \mu \mathrm{L}$ e a amostra foi diluída 10 vezes (1:10) em água antes de sua injeção.

\section{Resultados e Discussão}

\section{Análises físico-químicas}

Os resultados de caracterização físico-química da polpa de camu-camu são apresentados na Tabela 1. O camu-camu apresentou-se com teor de umidade alto, corroborando resultados encontrados por Aguiar e Souza (2015) e Fujita et al. (2013), 94,1 \pm 0,1 g/ $100 \mathrm{~g}$ de fruto e 92,9 $\pm 0,1 \mathrm{~g} / 100 \mathrm{~g}$ de camu-camu, respectivamente, conferindo uma característica suculenta a esse fruto.

Tabela 1. Caracterização físico-química da polpa congelada de camu-camu.

\section{Característica}

Umidade $(\mathrm{g} / 100 \mathrm{~g})$

Gorduras totais $(\mathrm{g} / 100 \mathrm{~g})$

Proteínas totais $(\mathrm{g} / 100 \mathrm{~g})$

Açúcares totais $(\mathrm{g} / 100 \mathrm{~g})$

Acidez total $(\mathrm{g} / 100 \mathrm{~g})$

$\mathrm{pH}$

Sólidos Solúveis ( ${ }^{\circ}$ Brix $)$

Ácido ascórbico $(\mathrm{mg} / 100 \mathrm{~g})$

\section{Média \pm Desvio padrão}

$$
92,77 \pm 0,15
$$

$0,14 \pm 0,01$

$0,35 \pm 0,07$

$8,00 \pm 0,28$

$1,93 \pm 0,01$

$2,93 \pm 0,01$

$6,2 \pm 0,00$

$2241 \pm 2,62$

O camu-camu apresentou baixo pH, que, segundo Nascimento et al. (2013), é explicado pelo teor de ácido ascórbico. Esse fato combinado com o baixo conteúdo de açúcares também comprova a sua baixa aceitação, mesmo pela população da região amazônica, e a sua pouca exploração comercial devido ao sabor extremamente ácido (MAEDA et al., 2006). 
Rufino et al. (2010) ao avaliarem diversas frutas tropicais do Brasil verificaram que o camu-camu apresentou o maior nível de vitamina C $(1882 \pm 43,2 \mathrm{mg} / 100 \mathrm{~g}$ de fruta fresca), sendo superior ao da acerola $(1357 \pm 9,5 \mathrm{mg} / 100 \mathrm{~g}$ de fruta fresca). Neste trabalho o conteúdo de vitamina $C$ foi superior ao encontrado por Baldeón et al. (2015) (1092,85 \pm 47,68 mg/100 $\mathrm{g}$ de fruta fresca), porém próximo ao encontrado por Aguiar e Souza (2015) (2031 \pm 0,04 $\mathrm{mg} / 100 \mathrm{~g}$ de fruta fresca) e Rufino et al. (2010).

Em um trabalho Grigio et al. (2016), em que foram avaliados o estágio de maturação e o tempo de estocagem da fruta, todos os estágios de maturação (imaturo, semi-maduro e maduro) e no tempo inicial assim como nos demais tempos de estocagem apresentaram teores de ácido ascórbico superiores ao deste trabalho, atingindo o nível de $6112 \mathrm{mg} / 100 \mathrm{~g}$ de fruta fresca, que os autores atribuíram ao fato de a polpa e a casca terem sido processadas juntas, verificando que a casca do camu-camu contribui com seu alto conteúdo de ácido ascórbico e é um subproduto que poderia ser utilizado pela indústria alimentícia para obtenção de vitamina C tanto para fortificar alimentos, quanto como conservante.

Determinação e quantificação de compostos fenólicos, análises de capacidade redutora e capacidade antioxidante

Os compostos fenólicos identificados por UPLC-MS/MS são apresentados na Tabela 2. Os fenólicos principais encontrados foram o ácido gálico, a epigalocatequina, o ácido clorogênico, a quercetina, a rutina, a miricetina e o kaempferol.

Tabela 2. Compostos fenólicos identificados e quantificados na polpa congelada de camucamu por UPLC-MS/MS.

\section{Compostos Concentração (mg/100 g fruta)}

Miricetina

Ácido Gálico

Catequina

Quercetina

Ácido Clorogênico

Rutina 
Kaempferol $\quad 0,182$

Cafeína $\quad 0,086$

Ácido Hidroxibenzóico $\quad 0,076$

Ácido Protocatecuíco $\quad 0,072$

Ácido Nicotínico $\quad 0,062$

Ácido p-Cumárico $\quad 0,054$

$\begin{array}{ll}\text { Teobromina } & 0,022\end{array}$

Epicatequina $\quad 0,018$

Ácido Sinápico $\quad 0,004$

Balisteiro et al. (2017) ao analisarem sucos de 6 frutas nativas do Brasil, dentre elas o camu-camu, verificaram que os compostos fenólicos encontrados por cromatografia líquida de alta eficiência acoplado a um detector de arranjo de diodos (HPLC/DAD) foram ácido siríngico, quercetina, ácido elágico livre, ácido elágico total e derivados de miricetina, não sendo identificados catequina, epicatequina e derivados do kaempferol encontrados neste trabalho. Os derivados de quercetina e de miricetina, os únicos compostos semelhantes com este trabalho, apresentaram valores de 0,46 e 0,61 mg/100 mL de suco, respectivamente, ou seja, a concentração de quercetina apresentou-se semelhante enquanto os níveis de miricetina foram inferiores aos deste estudo.

Fracassetti et al. (2013) ao analisar a polpa, semente e casca do fruto fresco de camucamu, assim como a polpa em pó e farinha por cromatografia líquida de alta eficiência acoplado a um detector de arranjo de fotodiodos (HPLC/PDA) encontraram compostos derivados de quercetina, de miricetina, de ácido elágico, de ácido gálico, antocianinas, elagitaninos e proantocianinas. Entretanto, na polpa fresca apenas derivados de miricetina $(1,79 \mathrm{mg} / 100 \mathrm{~g}$ de polpa fresca), de quercetina $(0,14 \mathrm{mg} / 100 \mathrm{~g}$ de polpa), do ácido elágico $(0,06 \mathrm{mg} / 100 \mathrm{~g}$ de polpa), antocianinas $(0,32 \mathrm{mg} / 100 \mathrm{~g}$ de polpa) e elagitaninos $(6,67 \mathrm{mg} / 100 \mathrm{~g}$ de polpa) foram encontrados, sendo os níveis de derivados de miricetina e de quercetina inferiores aos níveis de quercetina e miricetina deste estudo.

Essas diferenças nos níveis e tipos de compostos fenólicos podem ser devido ao estágio de maturação, região de cultivo e também a apresentação da fruta, pois a polpa congelada apresentou maior conteúdo desses compostos do que o camu-camu fresco em um estudo que 
realizou a composição química da polpa congelada e seus efeitos no perfil lipídico, glicemia, capacidade antioxidante, peroxidação lipídica, e atividade antioxidante de enzima no plasma de roedores com diabetes do tipo 1 , sendo possível observar melhora na hiperlipidemia e peroxidação lipídica desses ratos (GONÇALVES et al., 2014).

A capacidade redutora apresentada na Tabela 3 foi superior aos valores encontrados por Chirinos et al. (2010), $1120 \pm 47,1420 \pm 193$ e $1320 \pm 102$ mg EAG em 100 gramas de fruta fresca para os estágios de maturação: totalmente verde, verde-avermelhado e vermelho, respectivamente. Porém, a fruta estudada era proveniente do Peru, enquanto a utilizada nesse estudo foi cultivada no Amazonas. Esses autores concluíram que o camu-camu apresentou alta capacidade redutora ao compará-lo com cerejas, ameixas, morangos, mirtilo, entre outras frutas do tipo berries.

Tabela 3. Capacidade redutora e capacidade antioxidante da polpa congelada de camu-camu.

Parâmetro

\section{Média \pm Desvio padrão}

Capacidade redutora (mg EAG/100g)

$\mathrm{DPPH}(\mu \mathrm{mol}$ trolox/g)

$\operatorname{ABTS}(\mu \mathrm{mol}$ trolox/g)

$\operatorname{FRAP}\left(\mu \mathrm{mol} \mathrm{Fe}_{2} \mathrm{SO}_{4} / \mathrm{g}\right)$
$2606,27 \pm 147,71$

$$
57,30 \pm 4,19
$$

$167,36 \pm 8,42$

$1133,03 \pm 151,44$

Neves et al. (2017) encontraram valores de capacidade redutora e ácido ascórbico de $12420,12 \mathrm{mg} \mathrm{GAE} / 100 \mathrm{~g}$ de fruta fresca e $3474,09 \mathrm{mg} / 100 \mathrm{~mL}$ de polpa, respectivamente, para frutos no início da maturação, sendo o melhor tempo de colheita para obtenção de maior quantidade desses compostos, pois com o amadurecimento a concentração deles diminuiu devido a reações catabólicas desse processo. Mesmo a colheita sendo feita no início da maturação, após 5 dias de estocagem a frio foram observadas perdas de 75,23 e 98,36\% para composto fenólicos e vitamina C, respectivamente (NEVES et al., 2017). A importância de se definir quando as perdas desses compostos ocorrem é devido a suas capacidades antioxidantes (CARVALHO-SILVA et al., 2014).

É possível observar na análise de DPPH deste trabalho que o camu-camu apresentou capacidade antioxidante inferior aos encontrados por Chirino et al. (2010) para três estágios de 
maturação analisados pelos autores $(153 \pm 8,185 \pm 11$ e $167 \pm 11 \mu \mathrm{mol}$ trolox/g para os estágios verde, verde-avermelhado e vermelho, respectivamente), o que assim como a capacidade redutora, pode ser devido a origem da fruta, uma vez que as frutas utilizadas neste estudo possuíam grau de maturação vermelho, ou seja, maduro.

A capacidade antioxidante obtida pelo método do ABTS foi superior a encontrada por

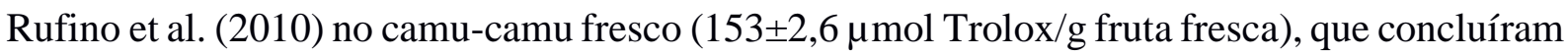
que, dentre as 18 frutas brasileiras analisadas, o camu-camu foi a que apresentou maior capacidade antioxidante, considerando tanto em matéria seca quanto fresca.

Azevedo et al. (2018) verificaram uma alta capacidade antioxidante, determinada por DPPH e ABTS, de frutos de camu-camu cultivados em ambientes seco $(1520,45 \pm 112,79$ e $1418,25 \pm 17,65 \mu \mathrm{mol}$ de equivalente de Trolox/g de camu-camu no estágio maduro para DPPH e ABTS, respectivamente) e inundado (1124,37 $\pm 29,12$ e 1209,08 $\pm 65,66 \mu$ mol de equivalente de Trolox/g de camu-camu no estágio maduro para DPPH e ABTS, respectivamente), sendo maior naqueles cultivados em ambiente seco e no estágio maduro, que concluíram que, mesmo não sendo cultivado em regiões nativas, esse fruto preserva sua capacidade antioxidante, a qual também pode estar mais relacionada aos conteúdos de antocianinas e polifenóis totais, presentes em maior quantidade nos frutos maduros.

O valor encontrado pelo método de FRAP assemelha-se ao apresentado por Chagas et al. (2015) para o camu-camu nos estágios de maturação "imaturo" e "semi-maturo" (1287,56 e 1355,56 $\mu \mathrm{mol} \mathrm{Fe2SO4/g,} \mathrm{respectivamente),} \mathrm{porém} \mathrm{com} \mathrm{valor} \mathrm{inferior} \mathrm{ao} \mathrm{estágio} \mathrm{"maturo"}$ (1994,93 $\mu \mathrm{mol}$ Fe2SO4/g). Desta forma, verifica-se que o estágio de maturação influência nos compostos presentes no camu-camu, que conferem a capacidade antioxidante dele. Ainda no referido trabalho os frutos foram provenientes de plantas nativas das margens do Lago da Morena no Estado de Roraima, diferentemente dos deste estudo que foram colhidos de plantas nativas das margens do Rio Madeira no Amazonas.

Uma alternativa para o sabor amargo e ácido do camu-camu é a produção de extrato em pó a fim de que o camu-camu possa ser utilizado como ingrediente pela indústria alimentícia com o papel de aumentar o conteúdo de antioxidantes de outros alimentos tanto visando a obtenção de um produto funcional quanto o utilizando como conservante. Entretanto, Azevêdo et al. (2014) observaram que o calor utilizado para secar os resíduos de camu-camu diminuiu consideravelmente o conteúdo de vitamina $\mathrm{C}$ e a sua capacidade redutora e, consequentemente, reduziu a capacidade antioxidante por meio do método de DPPH, tendo as perdas de capacidade redutora atingido 50,7 e 63,9\% e do ácido ascórbico 98,21 e 98,89\% para temperaturas de 40 e $80^{\circ} \mathrm{c}$, respectivamente. 
Quando o processo de secagem utilizado foi a liofilização, as perdas foram de 42,21\% e 97,42\% para capacidade redutora e ácido ascórbico, respectivamente. Logo, a polpa fresca congelada, como a utilizada neste estudo, conserva os compostos bioativos presentes no camucamu, por se tratar de um produto menos processado e armazenado em baixas temperaturas, sendo mais interessante para sua exploração considerando os benefícios para a saúde.

\section{Conclusões}

O camu-camu possui significantes teores de compostos bioativos e capacidade antioxidante, sendo uma fruta que poderia ser mais explorada no Brasil, porém há necessidade de se encontrar formas de consumi-la, o que aumentaria seu valor no mercado nacional e constituiria uma fonte de renda para produtores, podendo ser cultivada em locais diferentes da Amazônia, mas com o apelo de um produto brasileiro com propriedades funcionais.

\section{Referências}

AGUIAR J. P. L.; SOUZA F. C. A. Antioxidants chemical composition and minerals in freeze-dried camu-camu (Myrciaria dubia (H. B. K.) Mc Vaugh) pulp. Food and Nutrition Sciences, n. 6, p. 869-674, 2015.

AGUIAR, J. P. L.; SOUZA, F. DAS C. DO A. Antioxidant capacidant and bioactive compounds and health benefits of camu-camu puree (myrciaria dubia (h.b.k) mc vaugh).

International Journal of Development Research, v. 8, n. 6, p. 20742-20745, 2018.

AKACHI, T.; SHIINA, Y.; KAWAGUCHI, T.; KAWAGISHI, H.; MORITA, T.;

SUGIYAMA, K. 1-Methylmalate from camu-camu (myrciaria dubia) suppressed Dgalactosamine-induced liver injury in rats. Bioscience, Biotechnology and Biochemistry, v. 74, n. 3, p. 573-578, 2010.

AOAC. Association of official analytical chemists. Official methods of analysis of the AOAC International. 19 ed. Arlington, 2012.

AZEVÊDO, J. C. S.; BORGES, K. C.; GENOVESE, M. I.; CORREIA, R. T. P.; VATTEM, D. A. Neuroprotective effects of dried camu-camu (Myrciaria dubia HBK McVaugh) residue in C. elegans. Food Research International, v. 73, p. 135-141, 2015.

AZEVEDO, L.; DE ARAUJO RIBEIRO, P. F.; DE CARVALHO OLIVEIRA, J. A.; CORREIA, M. G.; RAMOS, F. M.; DE OLIVEIRA, E. B.; BARROS, F.; STRINGHETA, P. C. Camu-camu (Myrciaria dubia) from commercial cultivation has higher levels of bioactive compounds than native cultivation (Amazon Forest) and presents antimutagenic effects in vivo. Journal of the Science of Food and Agriculture, v. 99, n. 2, p. 624-631, 2019.

BALDEÓN, E. O.; ALCAÑIZ, M.; MASOT, R.; FUENTES, E. M.; BARAT, J. M.; GRAU, R. Voltammetry pulse array developed to determine the antioxidant activity of camu-camu 
(Myrciaria dubia (H.B.K.) McVaug) and tumbo (Passiflora mollisima (Kunth) L.H. Bailey) juices employing voltammetric electronic tongues. Food Control, v. 54, p. 181-187, 2015.

BALISTEIRO, D. M.; ARAUJO, R. L. De; GIACAGLIA, L. R.; GENOVESE, M. I. Effect of clarified Brazilian native fruit juices on postprandial glycemia in healthy subjects. Food Research International, v. 100, p. 196-203, 2017.

BARADARAN, A.; NASRI, H.; RAFIEIAN-KOPAEI, M. Oxidative stress and hypertension: Possibility of hypertension therapy with antioxidants. Journal of Research in Medical Sciences, v. 19, p. 358-367, 2014.

CARVALHO-SILVA, L. B.; DIONÍSIO, A. P.; PEREIRA, A. C. S.; WURLITZER, N. J.; BRITO, E. S. De; BATAGLION, G. A.; BRASIL, I. M.; EBERLIN, M. N.; LIU, R. H. Antiproliferative, antimutagenic and antioxidant activities of a Brazilian tropical fruit juice. LWT - Food Science and Technology, v. 59, n. 2P2, p. 1319-1324, 2014.

CHAGAS E. A.; GRIGIO M. L.; DURIGAN M. F. B.; FUJITA E.; VIEITES R. L. Caracterização centesimal e compostos bioativos de frutos do camu-camu em diferentes estádios de maturação. In: $1^{\circ}$ Congresso Brasileiro de Processamento Mínimo e Pós-colheita de Frutas, Flores e Hortaliças, 2015, Aracaju - SE. Anais do Congresso Brasileiro de Processamento Mínimo e Pós-colheita de Frutas, Flores e Hortaliças, 2015.

CHIRINOS, R.; GALARZA, J.; BETALLELUZ-PALLARDEL, I.; PEDRESCHI, R.; CAMPOS, D. Antioxidant compounds and antioxidant capacity of Peruvian camu camu (Myrciaria dubia (H.B.K.) McVaugh) fruit at different maturity stages. Food Chemistry, v. 120, n. 4, p. 1019-1024, 2010.

CONCEIÇÃO, N. et al. By-products of camu-camu [Myrciaria dubia (Kunth) McVaugh] as promising sources of bioactive high added-value food ingredients: Functionalization of yogurts. Molecules, v. 25, n. 1, p. 1-17, 2020.

CUNHA-SANTOS, E. C. E. et al. Vitamin C in camu-camu [Myrciaria dubia (H.B.K.) McVaugh]: evaluation of extraction and analytical methods. Food Research International, v. 115 , p. 160-166, 2019.

DE AZEVÊDO, J. C. S.; FUJITA, A.; DE OLIVEIRA, E. L.; GENOVESE, M. I.; CORREIA, R. T. P. Dried camu-camu (Myrciaria dubia H.B.K. McVaugh) industrial residue: A bioactive-rich Amazonian powder with functional attributes. Food Research International, v. 62, p. 934-940, 2014.

EMBRAPA. A cultura do camu-camu. Embrapa Amazônia Oriental. Brasília: Embrapa, 2012. $81 \mathrm{p}$.

FRACASSETTI, D.; COSTA, C.; MOULAY, L.; TOMÁS-BARBERÁN, F. A. Ellagic acid derivatives, ellagitannins, proanthocyanidins and other phenolics, vitamin $\mathrm{C}$ and antioxidant capacity of two powder products from camu-camu fruit (Myrciaria dubia). Food Chemistry, v. 139 , p. 578-588, 2013.

FUJTA, A. et al. Improving anti-hyperglycemic and anti-hypertensive properties of camucamu (Myriciaria dubia Mc. Vaugh) using lactic acid bacterial fermentation. Process 
Biochemistry, v. 59, p. 133-140, 2017.

FUJITA, A.; BORGES, K.; CORREIA, R.; FRANCO, B. D. G. de M.; GENOVESE, M. I. Impact of spouted bed drying on bioactive compounds, antimicrobial and antioxidant activities of commercial frozen pulp of camu-camu (Myrciaria dubia Mc. Vaugh). Food Research International, v. 54, p. 495-500, 2013.

GONÇALVES, A. E. D. S. S.; LELLIS-SANTOS, C.; CURI, R.; LAJOLO, F. M.; GENOVESE, M. I. Frozen pulp extracts of camu-camu (Myrciaria dubia McVaugh) attenuate the hyperlipidemia and lipid peroxidation of Type 1 diabetic rats. Food Research International, v. 64, p. 1-8, 2014.

GRIGIO, M. L.; CHAGAS, E. A.; BERLINGIERI DURIGAN, M. F.; DE ANDRADE SOUSA, A.; MOTA FILHO, A. B.; CHAGAS, P. C. Determination of harvest time and quality of native camu-camu fruits (Myrciaria dubia (Kunth) Mc Vaugh) during storage. Fruits, v. 71, n. 6, p. 373-378, 2016.

INOUE, T.; KOMODA, H.; UCHIDA, T.; NODE, K. Tropical fruit camu-camu (Myrciaria dubia) has anti-oxidative and anti-inflammatory properties. Journal of Cardiology, v. 52, p. 127-132, 2008.

INSTITUTO ADOLFO LUTZ (IAL). Métodos físico-químicos para análise de alimentos. São Paulo: Instituto Adolfo Lutz, 2008.

INSTITUTO NACIONAL DE PESQUISAS DA AMAZÔNIA (INPA). Cultivo do camucamu. Disponível em: www.inpa.gov.br/cpca/areas/camu-camu.html. Acesso em: 31 julho 2020.

KALLAUR A. P.; OLIVEIRA S. R.; REICHE E. M. V. The role of genetic polymorphisms in oxidative stress. In.: DICHI J.; BREGANÓ J. W.; SIMÃO A. N. C.; CECCHINI R. (Ed.). New York: CRC Press, 2014, p. 7-44.

KANESHIMA, T. et al. Antimicrobial constituents of peel and seeds of camu-camu (Myrciaria dubia). Bioscience, Biotechnology and Biochemistry, v. 81, n. 8, p. 1461-1465, 2017.

LANGLEY, P. C.; PERGOLIZZI, J. V.; TAYLOR, R.; RIDGWAY, C. Antioxidant and associated capacities of camu camu (Myrciaria dubia): A systematic review. Journal of Alternative and Complementary Medicine, v. 21, n. 1, p. 8-14, 2015.

MAEDA, R. N.; PANTOJA, L.; YUYAMA, L. K. O.; CHAAR, J. M. Determinação da formulação e caracterização do néctar de camu-camu (Myrciaria dubia McVaugh). Ciência e Tecnologia de Alimentos, v. 26, n. 1, p. 70-74, 2006.

NASCIMENTO, O. V.; BOLETI, A. P. A.; YUYAMA, L. K. O.; LIMA, E. S. Effects of diet supplementation with Camu-camu (Myrciaria dubia HBK McVaugh) fruit in a rat model of diet-induced obesity. Anais da Academia Brasileira de Ciências, v. 85, n. 1, p. 355-363, 2013.

NEVES, L. C.; DE CAMPOS, A. J.; CISNEROS-ZEVALLOS, L.; COLOMBO, R. C.; 
ROBERTO, S. R. Postharvest behavior of camu-camu fruits based on harvesting time and nutraceutical properties. Scientia Horticulturae, v. 217, p. 276-284, 2017.

NIMSE, S. B.; PAL, D. Free radicals, natural antioxidants, and their reaction mechanisms. RSC Advances, v. 5, n. 35, p. 27986-28006, 2015.

REYNERTSON, K. A.; YANG, H.; JIANG, B.; BASILE, M. J.; KENNELLY, E. J. Quantitative analysis of antiradical phenolic constituents from fourteen edible Myrtaceae fruits. Food Chemistry, v. 109, n. 4, p. 883-890, 2008.

RIBEIRO S. I.; MOTA M. G. C.; CORREAA M. L. P. Recomendações para o cultivo do camucamuzeiro no Estado do Pará. Belém: Embrapa, 2002.

RUFINO, M. do S. M.; ALVES, R. E.; DE BRITO, E. S.; PÉREZ-JIMÉNEZ, J.; SAURACALIXTO, F.; MANCINI-FILHO, J. Bioactive compounds and antioxidant capacities of 18 non-traditional tropical fruits from Brazil. Food Chemistry, v. 121, n. 4, p. 996-1002, 2010.

RUFINO, M. S. M.; ALVES, R. E.; FERNANDES, F. A. N.; BRITO, E. S. Free radical scavenging behavior of ten exotic tropical fruits extracts. Food Research International, v. 44, n. 7, p. 2072-2075, 2011.

SINGLETON V. L.; ROSSI J. A. Colorimetry of total phenolics with phosphomolybdicphosphotungstic acid reagents. American Journal of Enology and Viticulture, v. 16, n. 3, p. 144-158, 1965.

SOUZA FILHO, M. et al. Physico-chemical and Sensory Characterization of Nectars from Native Fruits from the North and Northeast of Brazil: Exploratory study. Brazilian Journal of Food Technology, v. 5, p. 139-143, 2002.

VIDIGAL, M. C. T. R.; MINIM, V. P. R.; CARVALHO, N. B.; MILAGRES, M. P.; GONÇALVES, A. C. A. Effect of a health claim on consumer acceptance of exotic Brazilian fruit juices: Açaí (Euterpe oleracea Mart.), Camu-camu (Myrciaria dubia), Cajá (Spondias lutea L.) and Umbu (Spondias tuberosa Arruda). Food Research International, v. 44, n. 7, p. 1988-1996, 2011. 